\title{
PREDICCIÓN DE LAS FUNCIONES DE CONTENIDO VOLUMÉTRICO DE HUMEDAD Y CONDUCTIVIDAD HIDRÁULICA PARAALGUNOS SUELOS DE GRANO FINO DE COSTA RICA
}

\author{
PREDICTION OF VOLUMETRIC WATER CONTENT AND HYDRAULIC \\ CONDUCTIVITY FUNCTIONS FOR SOME FINE GRAINED SOILS OF COSTA RICA
}

\author{
Rolando Mora \\ Escuela Centroamericana de Geología, Universidad de Costa Rica \\ Apartado: 2-14-2060UCR \\ rmorach@geologia.ucr.ac.cr
}

(Recibido: 01/12/2009; aceptado: 22/06/2010)

\begin{abstract}
RESUMEN: Se presentan metodologías para la predicción de funciones de contenido volumétrico de humedad y conductividad hidráulica, las cuales se utilizan para caracterizar el comportamiento no saturado de cuatro muestras de suelos de grano fino, provenientes de diferentes partes del país y de diferentes formaciones geológicas. La definición de estas funciones es importante, pues son la base para poder modelar numéricamente los procesos de flujo del agua subterránea, transporte de contaminantes y el establecimiento de zonas de protección de pozos y manantiales, en los suelos estudiados. El avance que se ha alcanzado hasta este momento, en términos de de la predicción del comportamiento no saturado de suelos, permite ofrecer a la comunidad científica nacional un medio para el establecimiento de las funciones predictivas bastante confiable y realizable en un período de tiempo corto.

Palabras clave: hidrogeología, conductividad hidráulica, flujo subterráneo, transporte de contaminantes, zona no saturada, contenido de humedad, succión.
\end{abstract}

\begin{abstract}
Methodologies for the prediction of functions of volumetric water content and hydraulic conductivity are introduced, which are used to characterize the non saturated behavior of four fine grained soils from different parts of the country and from different geological formations. The definition of these functions is important because they are the base to numerically model processes of groudwater flow, transport of pollutants and the establishment of protection areas around wells and springs, in the studied soils. The advance that has been reached until this moment, in terms of prediction of the un-saturated behavior of soils, allows offering to the national scientific community a quite reliable means for the establishment of the functions and it can be made in a short period of time.

Keywords: hydrogeology, hydraulic conductivity, groundwater flow, contaminant transport, partially saturated zone, moisture content, suction.
\end{abstract}




\section{INTRODUCCIÓN}

El análisis de los procesos de flujo subterráneo saturado-no saturado requiere del establecimiento de la relación existente entre la conductividad hidráulica y la presión del agua en los poros del material. En el caso de flujo transiente también se debe definir la función del contenido volumétrico de humedad. Estas dos funciones se pueden medir directamente en el laboratorio o se pueden predecir utilizando varios métodos. La función del contenido volumétrico de humedad se puede predecir de la curva de distribución granulométrica y la función de la conductividad hidráulica puede establecerse usando la función del contenido volumétrico de humedad y la conductividad hidráulica saturada medida.

Las dificultades que se presentan a la hora de medir directamente la función de conductividad hidráulica no saturada, se pueden superar mediante la predicción de esta a partir de una función de contenido volumétrico de humedad medida o predicha. En consecuencia, este es el abordaje preferido, si se cuenta con modelos predictivos apropiados.

No es difícil realizar una medida directa de la función de contenido volumétrico de humedad en el laboratorio, pero se requiere tiempo y encontrar un laboratorio de suelos que brinde el servicio. Por otro lado, el ensayo granulométrico es sencillo de realizar y existen muchos laboratorios que lo pueden ejecutar. Por lo tanto, es muy oportuno el desarrollo de un proceso de estimación de la función de contenido volumétrico de humedad, mediante soluciones que requieran parámetros de ajuste de curvas o curvas de distribución granulométrica.

En este trabajo se utiliza un método para predecir la función de la conductividad hidráulica no saturada, de algunos suelos de Costa Rica, utilizando una función estimada de contenido volumétrico de humedad y conductividades hidráulicas saturadas medidas en el campo. Lo anterior permite establecer los pasos necesarios para obtener estas funciones, las cuales son determinantes a la hora de realizar modelos numéricos para evaluar el flujo de aguas subterráneas, el transporte de contaminantes o establecer áreas de protección alrededor de pozos y manantiales.

\section{MÉTODO UTILIZADO PARA PREDECIR EL CONTENIDO VOLUMÉTRICO DE HUMEDAD}

Aubertin et al. (2001) presentan un método para predecir la función del contenido volumétrico de humedad, el cual es una modificación del método propuesto por Kovacs (1981). Las modificaciones al método implican que su uso se extienda a suelos de grano fino. El método predice la función del contenido volumétrico de humedad usando propiedades básicas del material.

La función es inicialmente determinada como una función del grado de saturación y posteriormente convertida a una función del contenido volumétrico de humedad. La función se desarrolla definiendo el grado de saturación para dos componentes principales. El primero se refiere al contenido de humedad que es almacenado en el suelo por las fuerzas capilares $\left(\mathrm{S}_{\mathrm{c}}\right)$. El segundo considera la función del contenido volumétrico de humedad a grandes presiones de poro negativas, donde el contenido de humedad existente en el suelo es, principalmente, una función de la adhesión $\left(\mathrm{S}_{\mathrm{a}}\right)$. Estos dos componentes $\left(\mathrm{S}_{\mathrm{c}}\right.$ y $\left.\mathrm{S}_{\mathrm{a}}\right)$ se pueden evaluar a partir de la presión de poro negativa e información sobre propiedades del material como el tamaño de grano, la forma de las partículas y la porosidad.

El grado de saturación $\left(\mathrm{S}_{\mathrm{r}}\right)$ se determina con base en la capilaridad $\left(\mathrm{S}_{\mathrm{c}}\right.$ ) y el componente adhesivo $\left(\mathrm{S}_{\mathrm{a}}\right)$ de la siguiente manera (Aubertin et al., 2001):

$$
\mathrm{S}_{\mathrm{r}}=\frac{\theta}{n}=S_{c}+S_{a} *\left(1-S_{c}\right)
$$

donde:

$\mathrm{S}_{\mathrm{r}}=$ grado de saturación

$\theta=$ contenido volumétrico de humedad

$\mathrm{n}=$ porosidad

$\mathrm{S}_{\mathrm{c}}=$ grado de saturación debido a las fuerzas capilares

$\mathrm{S}_{\mathrm{a}}^{*}=$ valor calculado a partir del grado de saturación debido a la adhesión $\left(\mathrm{S}_{\mathrm{a}}\right)$ (Aubertin et al., 2001): 


$$
S_{a} *=1-\left(1-S_{a}\right)
$$

El componente adhesivo es un valor calculado, pues es posible que a succiones bajas el valor de $\mathrm{S}_{\mathrm{a}}$ sea mayor que 1. El valor calculado asegura que para un Sa mayor o igual a $1, \mathrm{~S}_{\mathrm{a}}^{*}=1$ y si $\mathrm{S}_{\mathrm{a}}$ es menor que 1, entonces $\mathrm{S}_{\mathrm{a}}^{*}=\mathrm{S}_{\mathrm{a}}$.

El componente adhesivo $\left(\mathrm{S}_{\mathrm{a}}\right)$ se asocia con la capa delgada de agua que cubre la superficie de los granos del suelo y depende de propiedades básicas del material como la presión de poro negativa, el tamaño de grano, el coeficiente de forma y la porosidad del suelo. Se determina con la siguiente ecuación (Aubertin et al., 2001):

$$
S_{a}=a_{c} C_{\psi} \frac{\left(h_{c o} / \psi_{n}\right)^{2 / 3}}{e^{1 / 3}\left(\psi / \psi_{n}\right)^{1 / 6}}
$$

donde:

$\mathrm{a}_{\mathrm{c}}=$ un parámetro de ajuste

$\psi=$ presión de poro negativa o succión

$\psi_{\mathrm{n}}=$ término de succión incluido para garantizar componentes adimensionales

e $=$ relación de vacíos

$\mathrm{h}_{\mathrm{co}}=$ el ascenso capilar promedio, determinado a partir de la siguiente ecuación (Aubertin et al., 2001):

$$
h_{c o}=\frac{b}{e D_{10}}
$$
2001):

para suelos capilares, ó (Aubertin et al.,

$$
h_{c o, P}=\frac{\xi}{e} w_{L}^{1.45}
$$

para suelos cohesivos.

$\mathrm{D}_{10}=$ el diámetro de partícula (en $\mathrm{cm}$ ) correspondiente al $10 \%$ que pasa en una curva de distribución granulométrica.

b (Aubertin et al., 2001):

$$
b\left(\mathrm{~cm}^{2}\right)=\frac{0,75}{1,17 \log \left(C_{U}\right)+1}
$$

$\mathrm{C}_{\mathrm{u}}=$ el coeficiente de uniformidad

$\mathrm{W}_{\mathrm{L}}=$ el límite líquido (\%)

$\xi=$ una constante aproximadamente igual a $402,2 \mathrm{~cm}^{2}$ (Aubertin et al., 2001).

$\mathrm{C} \psi=$ es un coeficiente de corrección que permite un descenso progresivo en el contenido de humedad a grandes succiones, forzando la función a $\theta_{\mathrm{w}}=0$ en $\psi_{0}=10^{6} \mathrm{kPa}$, y que fue inicialmente propuesto por Fredlund and Xing (1994) $\mathrm{y}$ descrito por la siguiente ecuación (Aubertin et al., 2001):

$$
C_{\psi}=1-\frac{\ln \left(1+\psi / \psi_{r}\right)}{\ln \left(1+\psi_{0} / \psi_{r}\right)}
$$

$\psi_{\mathrm{r}}=$ succión correspondiente al contenido residual de humedad $\left(\theta_{\mathrm{r}}\right)$, representa el punto donde un incremento posterior en la succión no causa una remoción efectiva de agua del suelo (Aubertin et al., 2001):

$$
\psi_{r}=0.86 h_{c o, P}{ }^{1.2}
$$

$$
\psi_{r}=0,86\left(\frac{\xi}{e}\right)^{1,2} w_{L}^{1,74}
$$

La saturación capilar, que depende esencialmente del diámetro de poro y la distribución de tamaños de poro, se expresa como sigue (Aubertin et al., 2001):

$$
S_{c}=1-\left[\left(h_{c o} / \psi\right)^{2}+1\right]^{n} \exp \left[-m\left(h_{c o} / \psi\right)^{2}\right]
$$

donde:

$\mathrm{m}=$ un parámetro de ajuste que considera la distribución de tamaños de poro y controla la forma y posición de la función de contenido volumétrico de humedad en la zona capilar. 
Para suelos cohesivos plásticos, con $\mathrm{h}_{\mathrm{co}, \mathrm{P}}$, el valor de los parámetro $\mathrm{m}$ y a puede ser tomado como constante con $\mathrm{m}=3,0 \mathrm{E} 10^{-5}$ y a $=7,0 \mathrm{E} 10^{-4}$ en las aplicaciones predictivas. Para suelos con base capilar, $m$ y a pueden tomarse como 1 y 0,01 respectivamente.

\section{MÉTODO UTILIZADO PARA PREDECIR LA CONDUCTIVIDAD HIDRÁULICA}

Green \& Corey (1971) presentan un método para predecir la conductividad hidráulica no saturada a partir de funciones características sueloagua y concluyen que su método es suficientemente exacto para la mayoría de las aplicaciones. La ecuación que usan es la siguiente (Green \& Corey, 1971):

$$
K(\theta)_{i}=\frac{K_{s}}{K_{s c}} \frac{30 \gamma^{2}}{\rho g \eta} \frac{\varepsilon^{p}}{n^{2}} \sum_{j=1}^{m}\left[(2 j+1-2 i) h_{j}^{-2}\right]
$$

donde:

$\mathrm{K}(\theta)_{\mathrm{i}}=$ la conductividad calculada para un contenido de humedad especificado o para una presión de poro negativa especificada $(\mathrm{cm} / \mathrm{min})$

$\mathrm{K}_{\mathrm{s}} / \mathrm{K}_{\mathrm{sc}}=$ conductividad hidráulica saturada medida / conductividad saturada calculada

$\mathrm{i}=$ la última clase de contenido de humedad en el final húmedo, $\mathrm{i}=1$ identifica el tipo de poro correspondiente al contenido de humedad mínimo e $\mathrm{i}=\mathrm{m}$ identifica el tipo de poro correspondiente al contenido de humedad saturado.

$\mathrm{h}_{\mathrm{j}}=$ la carga de presión de poro negativa para una clase dada de poros llenos de agua $(\mathrm{cm}$ de agua)

$\mathrm{n}=$ número total de tipos de poros entre i y $\mathrm{m}$

$\theta=$ contenido de humedad $\left(\mathrm{cm}^{3} / \mathrm{cm}^{3}\right)$

$\theta_{0}=$ contenido de humedad mínimo en la curva experimental $\left(\mathrm{cm}^{3} / \mathrm{cm}^{3}\right)$

$\theta_{\mathrm{s}}=$ contenido de humedad saturado $\left(\mathrm{cm}^{3} / \mathrm{cm}^{3}\right)$

$\gamma^{2}=$ tensión superficial del agua $(\mathrm{Dyn} / \mathrm{cm})$

$\varepsilon=$ porosidad saturada de agua

$\eta=$ viscosidad del agua $\left(\mathrm{g} / \mathrm{cm} \mathrm{s}^{-1}\right)$

$\mathrm{g}=$ constante gravitacional $\left(\mathrm{cm} / \mathrm{s}^{2}\right)$ $\mu=$ densidad del agua $\left(\mathrm{g} / \mathrm{cm}^{3}\right)$

$\mathrm{p}=$ un parámetro que considera la interacción de tipos de poros $(1<=\mathrm{p}<=2)$

\section{CURVAS GRANULOMÉTRICAS Y PROPIEDADES FÍSICAS E HIDRÁULICAS DE LOS SUELOS ESTUDIADOS}

La ubicación de las muestras utilizadas se presenta en la figura 1. Las curvas granulométricas de los suelos estudiados se presentan en la figura 2, su localización, clasificación y algunas propiedades físicas e hidráulicas, se pueden considerar en el cuadro 1. Se resalta que todas las muestras corresponden con suelos de grano fino, con conductividades hidráulicas saturadas bajas. Estas muestras se han seleccionado para este estudio, pues las mismas cuentan con todos los ensayos y datos requeridos para utilizar los métodos predictivos enunciados anteriormente. A continuación se desglosan los ensayos requeridos de las muestras para aplicar los métodos predictivos:

- Ensayo de distribución granulométrica

- Ensayo de conductividad hidráulica saturada de campo

- Ensayo de peso unitario húmedo

- Ensayo de límites de consistencia

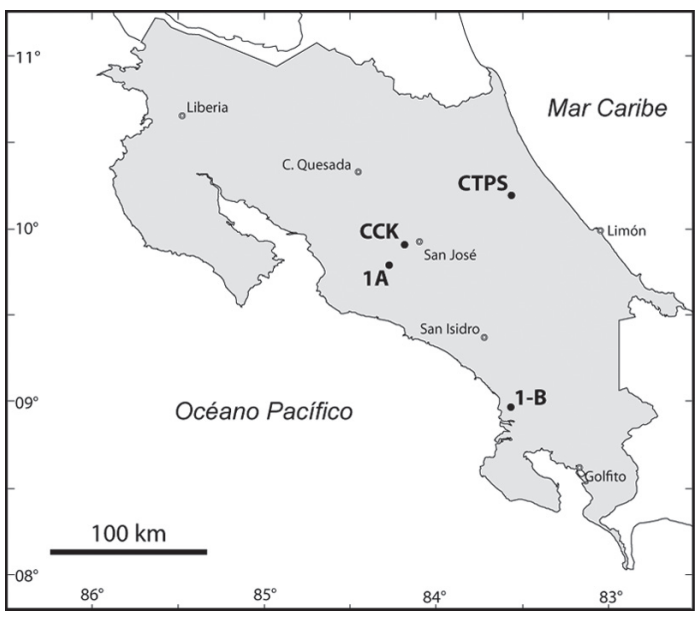

Fig. 1: Ubicación de las muestras analizadas 


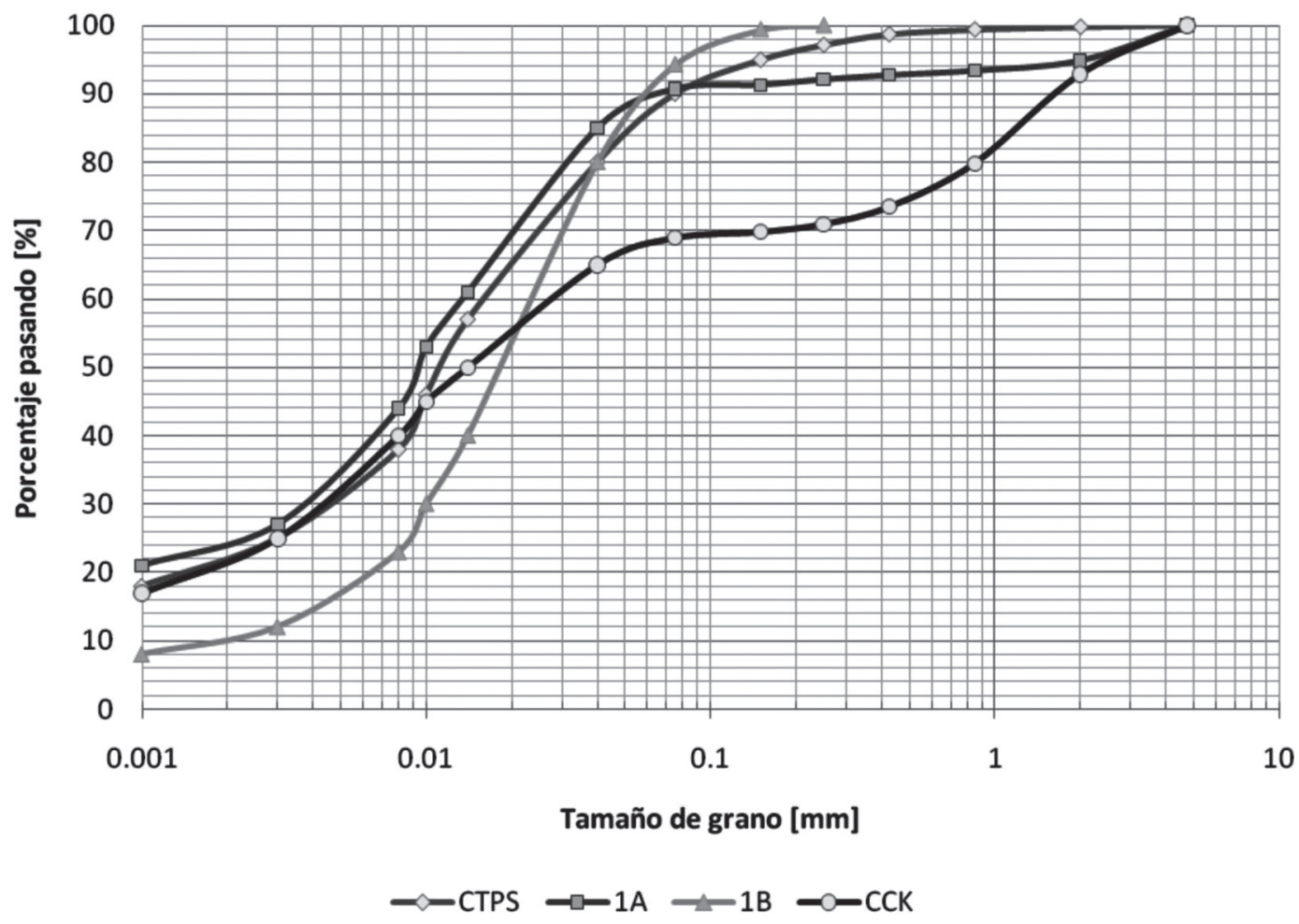

Fig. 2: Curvas granulométricas de los suelos estudiados

Cuadro 1

Localización y propiedades de los suelos estudiados

\begin{tabular}{|c|c|c|c|c|c|c|}
\hline Muestra & $\begin{array}{l}\text { Procedencia y } \\
\text { Fm. Geológica }\end{array}$ & Coordenadas & Clasificación SUCS & $\begin{array}{l}\text { Conductividad } \\
\text { hidráulica } \\
\text { saturada }[\mathrm{m} / \mathrm{s}]\end{array}$ & $\begin{array}{c}\text { Límite líquido } \\
{[\%]}\end{array}$ & Porosidad $[\%]$ \\
\hline CTPS & $\begin{array}{l}\text { Siquirres, Limón. } \\
\text { Depósitos Re- } \\
\text { cientes }\end{array}$ & $\begin{array}{l}230312 \mathrm{~N} \\
590011 \mathrm{E}\end{array}$ & $\begin{array}{c}\text { Limo elástico }(\mathrm{MH}) \\
\text { de plasticidad alta }\end{array}$ & $1,5 \mathrm{E}^{-06} \mathrm{~m} / \mathrm{s}$ & 75,2 & 62,1 \\
\hline $1 \mathrm{~A}$ & $\begin{array}{l}\text { Ococa, Guaitil, } \\
\text { Acosta, San José. } \\
\text { Fm. La Cruz }\end{array}$ & $\begin{array}{l}199370 \mathrm{~N} \\
514113 \mathrm{E}\end{array}$ & $\begin{array}{c}\text { Arcilla gruesa }(\mathrm{CH}) \\
\text { de } \\
\text { plasticidad alta }\end{array}$ & $\begin{array}{c}1,5 \mathrm{E}^{-06} \mathrm{~m} / \mathrm{s} \mathrm{a} \\
5,4 \mathrm{E}^{-07} \mathrm{~m} / \mathrm{s}\end{array}$ & 58,3 & 42,1 \\
\hline $1-B$ & $\begin{array}{c}\text { Sierpe, Osa, Pun- } \\
\text { tarenas. Depósitos } \\
\text { Recientes }\end{array}$ & $\begin{array}{l}314307 \mathrm{~N} \\
521526 \mathrm{~S}\end{array}$ & $\begin{array}{c}\text { Limo elástico }(\mathrm{MH}) \\
\text { de } \\
\text { plasticidad alta }\end{array}$ & $\begin{array}{c}2,1 \mathrm{E}^{-06} \mathrm{~m} / \mathrm{s} \mathrm{a} \\
2,0 \mathrm{E}^{-07} \mathrm{~m} / \mathrm{s}\end{array}$ & 50,9 & 54,6 \\
\hline $\mathrm{CCK}$ & $\begin{array}{l}\text { Escazú, San José. } \\
\text { Fm. Pacacua }\end{array}$ & $\begin{array}{l}210350 \mathrm{~N} \\
518800 \mathrm{E}\end{array}$ & $\begin{array}{l}\text { Arcilla delgada } \\
\text { (CI) de plasticidad } \\
\text { intermedia }\end{array}$ & $\begin{array}{c}3,0 \mathrm{E}^{-07} \mathrm{~m} / \mathrm{s} \mathrm{a} \\
3,1 \mathrm{E}^{-08} \mathrm{~m} / \mathrm{s}\end{array}$ & 35,0 & 38,0 \\
\hline
\end{tabular}


- Ensayo de contenido humedad natural

- Ensayo de gravedad específica

- Cálculo de la porosidad del material

\section{PREDICCIÓN DE LA FUNCIÓN DEL CONTENIDO VOLUMÉTRICO DE HUMEDAD}

Para cada muestra se ha realizado la predicción con el método modificado de Kovacs (Aubertin et al., 2003), mediante la utilización de una hoja de cálculo en donde se han incluido las ecuaciones necesarias para obtener los resultados y gráficos correspondientes. En la figura 3 se aprecian los resultados obtenidos para las cuatro muestras. Es notorio que el contenido de humedad en las cuatro muestras se mantiene inferior al $10 \%$ hasta cerca de una presión de $-40000 \mathrm{kPa}$, para incrementarse de manera súbita luego de este umbral y alcanzar el estado de saturación total.

\section{PREDICCIÓN DE LA FUNCIÓN DE LA CONDUCTIVIDAD HIDRÁULICA}

Para cada muestra se realiza la predicción con el método de Green \& Corey (1971), los resultados se pueden apreciar en la figura 4.

De la figura 4 se puede apreciar que los limos de plasticidad alta (muestras CTPS y 1-B) tienen funciones similares, cuando la conductividad hidráulica saturada es de alrededor de 10-06 m/s, si la conductividad hidráulica decrece la función se diferencia notablemente de las anteriores. En el caso de las arcillas se presenta una diferencia notoria entre las de plasticidad alta (1A) y las de plasticidad intermedia (CCK).

Es evidente que las funciones de la conductividad hidráulica son proporcionales a la conductividad hidráulica saturada medida, por lo tanto, se requiere realizar una campaña de muestreos y ensayos, con el objetivo de caracterizar estadísticamente este parámetro en los suelos de las diferen-

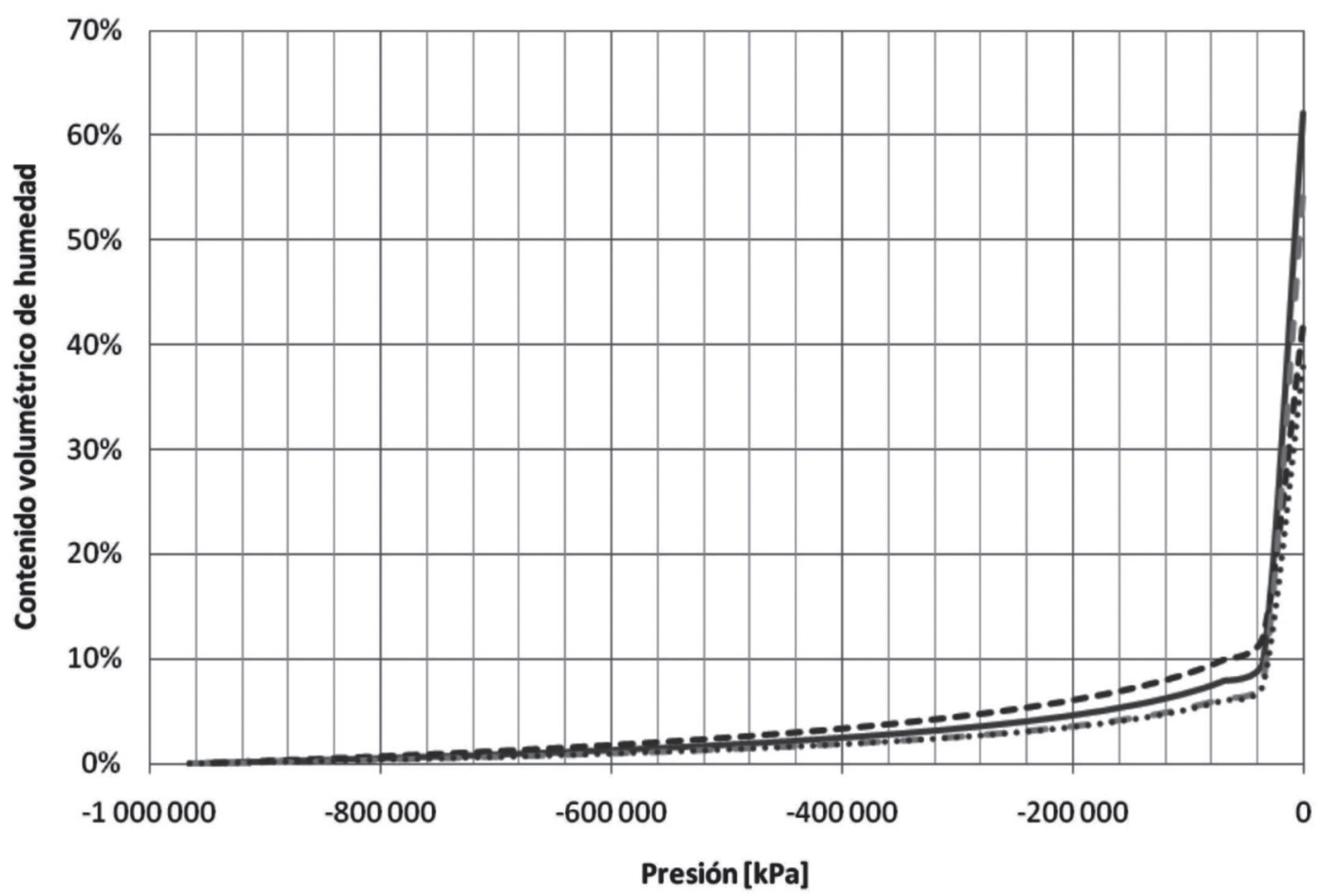

Fig. 3: Predicción de la función de contenido volumétrico de humedad de las muestras estudiadas 


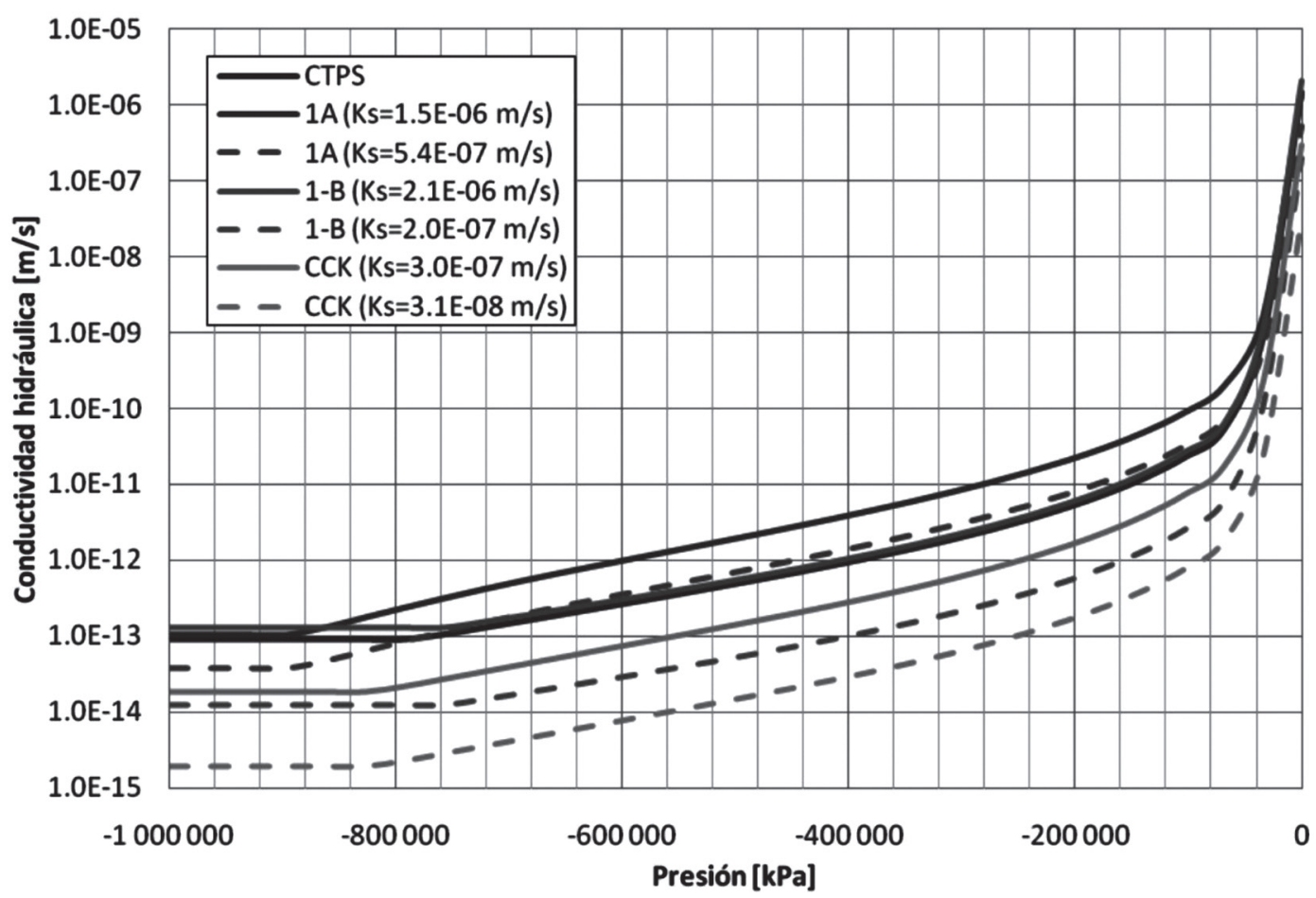

Fig. 4: Predicción de la función de la conductividad hidráulica de las muestras estudiadas

tes formaciones geológicas y así crear una base de datos que pueda utilizarse en la implementación de modelos de elementos finitos y diferencias finitas, para el estudio confiable del flujo de aguas subterráneas y el tránsito de contaminantes.

\section{CONCLUSIONES}

Debido a la carencia de laboratorios especializados en la medición de funciones de contenido volumétrico de humedad y conductividad hidráulica no saturada, en nuestro país se deben utilizar metodologías predictivas para aproximar estas funciones, a partir de ensayos de propiedades físicas fácilmente realizables. La utilización de funciones proporcionará una mejora sustancial de los resultados en el modelaje del flujo subterráneo en medios porosos y del transporte de contaminantes en este mismo medio.

Las cuatro muestras estudiadas presentan funciones de contenido volumétrico de humedad bastante similares, manteniendo un contenido volumétrico de humedad inferior al $10 \%$ hasta cerca de una presión de $-40000 \mathrm{kPa}$, para posteriormente incrementarse de manera súbita luego de este umbral y alcanzar el estado de saturación total. Este comportamiento se puede asociar al establecimiento del flujo de agua a través de una sección transversal de vacíos cada vez mayor, hasta alcanzar la saturación completa de la sección de vacios y así la conductividad hidráulica saturada.

Las funciones de conductividad hidráulica calculadas presentan diferencias importantes, así como, algunas coincidencias. Se requiere la realización de más ensayos de conductividad hidráulica saturada de campo y campañas de muestreo y ensayo de suelos, con el objetivo de caracterizar los suelos de la diferentes formaciones geológicas y así crear una base de datos que pueda utilizarse en la implementación de modelos de elementos finitos y diferencias finitas, para el estudio confiable del flujo de aguas subterráneas y el tránsito de contaminantes.

$\mathrm{El}$ avance que se ha alcanzado hasta este momento, en términos de de la predicción del comportamiento no saturado de suelos, permite ofrecer 
a la comunidad científica nacional un medio para el establecimiento de las funciones predictivas bastante confiable y realizable en un período de tiempo corto.

\section{REFERENCIAS}

AUBERTIN, M., MBONIMPA, M., BUSSIÈRE, B., CHAPUIS, R.P., 2003: A model to predict the water retention curve from basic geotechnical properties.- Canadian Geotechnical Journal, 40(6): 1104-1122
FREDLUND, D. G., \& ANQING XING, 1994: Equations for the soil-water characteristic curve.- Canadian Geotechnical Journal, Vol. 31: 521-532.

GREEN, R.E. AND COREY, J.C., 1971: Calculation of Hydraulic Conductivity: A Further Evaluation of Some Predictive Methods.- Soil Science Society of America Proceedings, 35: 3-8.

KOVÁCS, G., 1981: Seepage Hydraulic,-383 págs. Elsevier Science Publishers, Amsterdam. 